\title{
Trunk and lower limb muscularity in sprinters: what are the specific muscles for superior sprint performance?
}

\author{
Nobuaki Tottori ${ }^{1}$, Tadashi Suga ${ }^{2}$ (0), Yuto Miyake ${ }^{2}$, Ryo Tsuchikane ${ }^{2}$, Takahiro Tanaka², Masafumi Terada ${ }^{2}$, \\ Mitsuo Otsuka², Akinori Nagano ${ }^{2}$, Satoshi Fujita² and Tadao Isaka²
}

\begin{abstract}
Objective: The major purpose of this study was to determine the specific muscle(s) for superior sprint performance in sprinters. The cross sectional areas (CSAs) of ten muscles of the trunk and lower limb were measured using magnetic resonance images in 56 male sprinters and 40 male non-sprinters. In addition to the absolute CSA, to minimize the effect of difference in body size among participants, the relative CSA normalized to body mass was used for analysis of this study.

Results: Absolute and relative CSAs of most trunk and lower limb muscles, including the psoas major (PM) and gluteus maximus (GM), were significantly larger in sprinters than in non-sprinters (all $P<0.001, d=0.91$ to 1.82). The absolute and relative CSAs of the PM and GM correlated significantly with personal best 100-m sprint time in sprinters $(r=-0.363$ to -0.388 , all $P<0.01)$. A stepwise multiple regression analysis revealed that both CSAs of absolute PM and relative GM were predictive variables for the personal best $100 \mathrm{~m}$ sprint time in sprinters $(\beta=-0.289$ and -0.287 , respectively, both $P<0.05$ ). These findings suggest that the $P M$ and $G M$ may be specific muscles for superior sprint performance in sprinters.
\end{abstract}

Keywords: Cross-sectional area, Joint torque, Hip flexion, Hip extension, Magnetic resonance imaging

\section{Introduction}

The generation of large torques by muscles crossing the hip, knee, and ankle joints is necessary for superior sprint performance in sprinters [1]. The joint torque is largely determined by size of agonist muscle group [2]. Previous studies have reported that many muscles of the trunk and lower limb were greater in sprinters than in non-sprinters [3-5]. Furthermore, previous studies have determined that the sizes of some trunk and lower limb muscles are correlated with superior sprint performance in sprinters [6-12]. However, many of these studies have examined using only a few

\footnotetext{
*Correspondence: t-suga@fc.ritsumei.ac.jp

2 Faculty of Sport and Health Science, Ritsumeikan University, 1-1-1

Nojihigashi, Kusatsu, Shiga 525-8577, Japan

Full list of author information is available at the end of the article
}

muscles of the trunk and lower limb. Therefore, the specific muscles that contribute to superior sprint performance for sprinters have not been fully identified.

The contribution of the hip joint torque during sprinting is greater than that of the ankle and knee joint torques [1]; thus, greater hip muscles may play an important role for achieving better sprint performance in sprinters. The psoas major (PM) is a major muscle for the hip flexion. Several previous studies have reported that a greater PM size is correlated with better sprint performance in sprinters $[7,10,12]$. In addition, the gluteus maximus (GM) is a major muscle for the hip extensors. Sugisaki et al. reported that a greater GM size is correlated with better sprinter performance in sprinters [10]. However, the relationship between the GM size and sprint performance in sprinters remains poorly understood. 
To gain our understanding of specific muscle(s) for superior sprint performance in sprinters, this study first examined the differences in cross-sectional areas (CSA) of 10 selected muscles of the trunk and lower limb between sprinters and non-sprinters. Thereafter, we examined the relationships between the trunk and lower limb muscle CSAs and sprint performance in sprinters.

\section{Main text}

\section{Methods}

\section{Participants}

Fifty-six well-trained male sprinters (age: $20.7 \pm 1.6$ years) participated in this study. Their personal best time of a $100 \mathrm{~m}$ race time ranged from 10.32 to $11.80 \mathrm{~s}$ (mean, $11.12 \pm 0.36 \mathrm{~s}$ ) within the previous one year. They were involved in regular sprint training at least 5 times per week and had regularly competition. In addition, 40 non-sprinters (age: $21.1 \pm 1.1$ years) whose body size (i.e., body height and body mass) was similar to those of the sprinters were selected as a control group (see Table 1 ). The body sizematched control participants were recreationally active, but were not involved in any specific physical training program within the previous 3 years. Nevertheless, many of them had participated in recreational sports and/or physical training for 2-3 h per week. All participants were informed of the experimental procedures and provided written consent to participate in the study. This study was approved by the Ethics Committee of Ritsumeikan University.

\section{Magnetic resonance imaging (MRI)}

Representative MRI scans for measuring CSAs of the trunk and lower limb muscles are presented in Fig. 1. To avoid any effects from changes in muscle size due to heavy training, MRI measurements for sprinters were scheduled the following day after a rest day or light intensity training day during the off-season. Additionally, MRI measurements for sprinters who performed the light intensity training were scheduled at least $12 \mathrm{~h}$ after the training session. The MRI measurement has been described in our previous studies [11-13]. In brief, to measure the CSAs of the trunk and lower limb muscles, excluding the GM, participants were placed in a supine position on the scanner bed, with both knees fully extended and both ankles set at the neutral position (i.e., $0^{\circ}$ ). With regard to the trunk muscles, the CSAs of the rectus abdominis, lateral abdominal wall, erector spinae, and PM were obtained at the mid-level of the L4-L5 [7, 9, 11, 12]. With regard to the lower limb muscles, the CSAs of the adductors, quadriceps femoris (QF), hamstring (HAM), dorsiflexors (DF), and plantar

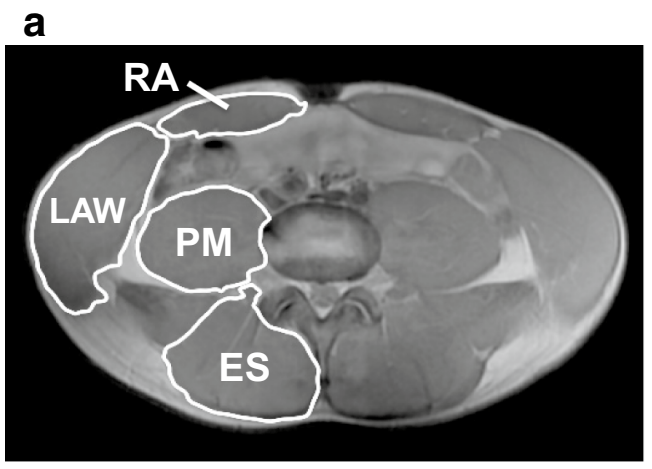

b

C

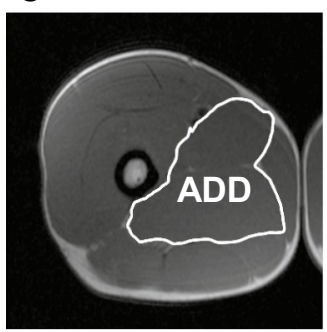

d

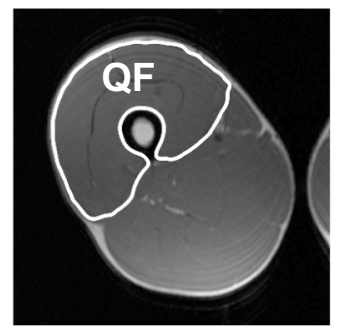

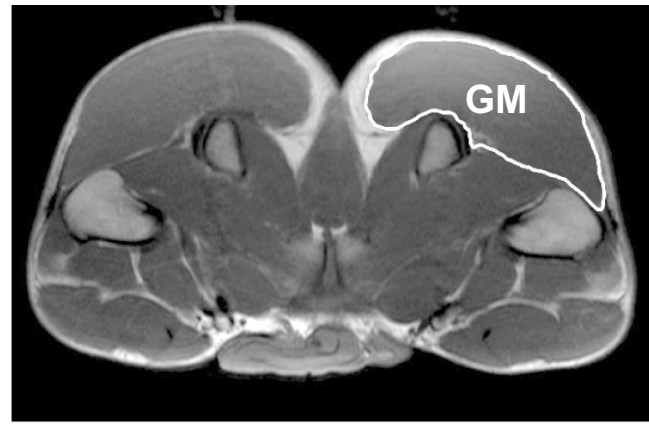

e

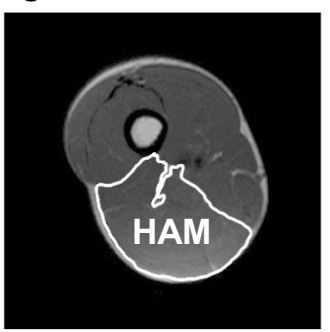

f

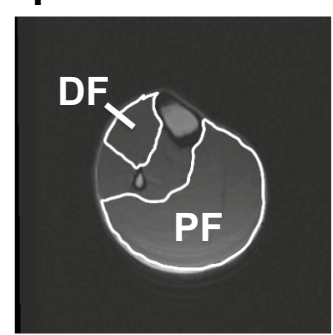

Fig. 1 Representative magnetic resonance imaging scans for measuring cross-sectional areas of trunk and lower limb muscles. The cross-sectional areas (CSA; CSAs) of the rectus abdominis (RA), lateral abdominal wall (LAW), elector spinae (ES), and psoas major (PM) were obtained at the mid-level of the L4-L5. a The CSA of the gluteus major (GM) was obtained at the level of the greater trochanter. $\mathbf{b}$ The CSA of the adductors (ADD) was obtained at the proximal $30 \%$ of the thigh length. c The CSA of the quadriceps femoris (QF) was obtained at $50 \%$ of the thigh length. d The CSA of the hamstrings (HAM) was obtained at 70\% of the thigh length. e The CSAs of the dorsiflexors (DF) and plantar flexors (PF) were obtained at the proximal $30 \%$ of the lower leg length (e) 
flexors were measured. The adductors CSA was obtained at the proximal $30 \%$ of the thigh length [9]. The QF CSA was obtained at the proximal $50 \%$ of the thigh length $[9,13]$. The HAM CSA was obtained at the proximal $70 \%$ of the thigh length [9]. The DF and plantar flexors CSAs were obtained at the proximal $30 \%$ of the lower leg length [9]. To measure the CSA of the GM, participants were placed in a prone position on the scanner, with both knees fully extended. The GM CSA was obtained at the level of the greater trochanter. The locations for calculating these trunk and lower limb CSAs were based on methods outlined in our and other previous studies $[7,9,11-13]$.

The CSAs of all ten muscles were calculated from the right side using image analysis software (OsiriX Version 5.6, Switzerland). In addition to the absolute CSA, to minimize the effects of difference in body size among participants, the relative CSA normalized to body mass to the two-thirds power was used for analysis of this study, as in our and other previous studies $[9,13]$.

\section{Statistical analysis}

Data are expressed as mean \pm SD. Comparisons of measured variables between sprinters and non-sprinters were performed using unpaired $t$-testing. The Cohen's $d$ effect size using the pooled SD was calculated to determine the magnitude of difference in outcome variables between the two groups. The magnitudes of the effect size were interpreted as small $(0.20 \leq d<0.50)$, medium $(0.50 \leq d<0.80)$ and large $(0.80 \leq d)$ [14]. The relationships between trunk and lower limb muscle CSAs and personal best 100-m sprint time in sprinters were evaluated using a Pearson's product moment correlation coefficient. A stepwise multiple regression analysis was used to determine the predictive variable(s) for the personal best 100 -m sprint time in sprinters using the absolute and relative CSAs as independent variables. Statistical significance level was defined at $P<0.05$. All statistical analyses were conducted using the IBM SPSS software (version 19.0; International Business Machines Corp, NY, USA).

\section{Results}

Physical characteristics and absolute and relative CSAs of the trunk and lower limb muscles in sprinters and nonsprinters are listed in Table 1. Body height and body mass did not differ significantly between sprinters and nonsprinters. The absolute and relative CSAs of most trunk and lower limb muscles, excluding the QF and DF, were

Table 1 Physical characteristics and absolute and relative cross-sectional areas (CSA) of trunk and lower limb muscles in sprinters and non-sprinters

\begin{tabular}{|c|c|c|c|c|}
\hline & Sprinters & Non-sprinters & $P$ value & Cohen's d \\
\hline Body height, $\mathrm{cm}$ & $175.4 \pm 5.1[174.0,176.8]$ & $174.6 \pm 4.7[173.2,176.0]$ & 0.613 & 0.16 \\
\hline Body mass, kg & $66.0 \pm 5.1[64.6,67.4]$ & $66.4 \pm 7.2[64.1,68.6]$ & 0.798 & 0.06 \\
\hline \multicolumn{5}{|l|}{ Absolute CSA, $\mathrm{cm}^{2}$} \\
\hline Rectus abdominis & $8.7 \pm 1.7[8.2,9.1]$ & $6.8 \pm 1.8[6.2,7.3]$ & $<0.001$ & 1.13 \\
\hline Lateral abdominal wall & $28.3 \pm 4.8[27.0,29.6]$ & $23.5 \pm 4.0[22.3,24.7]$ & $<0.001$ & 1.07 \\
\hline Erector spinae & $25.4 \pm 3.3[24.5,26.3]$ & $22.3 \pm 3.7[21.1,23.4]$ & $<0.001$ & 0.91 \\
\hline Psoas major & $20.5 \pm 2.7[19.8,21.3]$ & $16.9 \pm 2.5[16.1,17.6]$ & $<0.001$ & 1.40 \\
\hline Gluteus maximus & $60.1 \pm 7.2[58.2,62.0]$ & $50.8 \pm 5.8[49.0,52.6]$ & $<0.001$ & 1.41 \\
\hline Quadriceps femoris & $77.1 \pm 8.2[74.9,79.3]$ & $75.6 \pm 10.7[72.2,78.9]$ & 0.427 & 0.16 \\
\hline Hamstring & $46.8 \pm 6.7[45.0,48.6]$ & $37.7 \pm 7.2[35.5,40.0]$ & $<0.001$ & 1.31 \\
\hline Adductors & $58.2 \pm 6.3[56.5,59.9]$ & $49.4 \pm 8.4[46.8,52.0]$ & $<0.001$ & 1.22 \\
\hline Dorsiflexors & $10.9 \pm 1.2[10.6,11.2]$ & $10.6 \pm 1.5[10.2,11.1]$ & 0.367 & 0.20 \\
\hline Plantar flexors & $50.5 \pm 7.2[48.5,52.4]$ & $44.3 \pm 5.9[42.5,46.1]$ & $<0.001$ & 0.92 \\
\hline \multicolumn{5}{|l|}{ Relative CSA, $\mathrm{cm}^{2} / \mathrm{kg}^{2 / 3}$} \\
\hline Rectus abdominis & $0.53 \pm 0.09[0.51,0.56]$ & $0.41 \pm 0.10[0.38,0.44]$ & $<0.001$ & 1.26 \\
\hline Lateral abdominal wall & $1.73 \pm 0.27[1.66,1.80]$ & $1.43 \pm 0.21[1.37,1.50]$ & $<0.001$ & 1.21 \\
\hline Erector spinae & $1.56 \pm 0.18[1.51,1.60]$ & $1.36 \pm 0.18[1.30,1.42]$ & $<0.001$ & 1.10 \\
\hline Psoas major & $1.26 \pm 0.15[1.22,1.30]$ & $1.03 \pm 0.14[0.99,1.07]$ & $<0.001$ & 1.58 \\
\hline Gluteus maximus & $3.68 \pm 0.33[3.59,3.76]$ & $3.10 \pm 0.30[3.01,3.19]$ & $<0.001$ & 1.82 \\
\hline Quadriceps femoris & $4.72 \pm 0.42[4.61,4.83]$ & $4.60 \pm 0.45[4.46,4.74]$ & 0.192 & 0.27 \\
\hline Hamstring & $2.86 \pm 0.36[2.77,2.96]$ & $2.29 \pm 0.34[2.19,2.40]$ & $<0.001$ & 1.62 \\
\hline Adductors & $3.56 \pm 0.31[3.48,3.65]$ & $3.01 \pm 0.41[2.89,3.14]$ & $<0.001$ & 1.55 \\
\hline Dorsiflexors & $0.67 \pm 0.07[0.65,0.69]$ & $0.65 \pm 0.09[0.62,0.68]$ & 0.291 & 0.24 \\
\hline Plantar flexors & $3.09 \pm 0.37[2.98,3.19]$ & $2.70 \pm 0.29[2.61,2.79]$ & $<0.001$ & 1.12 \\
\hline
\end{tabular}

Values are presented as Mean \pm SD [the upper and lower limits of a $95 \%$ confidence interval]. The relative CSA was normalized to body mass to the two-thirds power. Bold values indicate significance differences $(P<0.05)$ between sprinters and non-sprinters 
significantly larger in sprinters than in non-sprinters (all $P<0.001)$, with large effect size $(d=0.91$ to 1.82$)$.

Correlation coefficients of absolute and relative CSAs of the trunk and lower limb muscles with personal best 100-m sprint time in sprinters are shown in Table 2. Among the ten muscles, the absolute and relative CSAs of only the PM and GM correlated significantly with personal best $100-\mathrm{m}$ sprint time in sprinters $(r=-0.363$ to -0.388 , all $P<0.01)$. A stepwise multiple regression analysis revealed that both CSAs of absolute PM and relative GM were predictive variables for the personal best $100-\mathrm{m}$ sprint time in sprinters $(\beta=-0.289$ and -0.287 , respectively, both $P<0.05)$. The adjusted $R^{2}$ for this analysis was $0.194(P=0.001)$.

\section{Discussion}

This study determined that the absolute and relative CSAs of most trunk and lower limb muscles, excluding the QF and DF, were larger in sprinters than in nonsprinters. In particular, absolute CSAs of the PM and GM was 21.7 and $18.4 \%$, respectively, higher in sprinters than Table 2 Correlation coefficients between absolute
and relative CSAs of trunk and lower limb muscles
and personal best 100-m sprint time in sprinters

$r$ [the upper and lower limit $P$ value of a $95 \% \mathrm{Cl}]$

\begin{tabular}{|c|c|c|}
\hline \multicolumn{3}{|l|}{ Absolute CSA } \\
\hline Rectus abdominis & $-0.009[-0.271,0.255]$ & 0.945 \\
\hline Lateral abdominal wall & $-0.200[-0.440,0.066]$ & 0.140 \\
\hline Erector spinae & $-0.119[-0.149,0.370]$ & 0.383 \\
\hline Psoas major & $-0.388[-0.591,-0.139]$ & 0.003 \\
\hline Gluteus maximus & $-0.366[-0.574,-0.114]$ & 0.006 \\
\hline Quadriceps femoris & $-0.040[-0.300,0.225]$ & 0.767 \\
\hline Hamstring & $-0.107[-0.360,0.160]$ & 0.434 \\
\hline Adductors & $-0.092[-0.347,0.175]$ & 0.502 \\
\hline Dorsiflexors & $0.088[-0.179,0.343]$ & 0.521 \\
\hline Plantar flexors & $-0.132[-0.382,0.136]$ & 0.332 \\
\hline \multicolumn{3}{|l|}{ Relative CSA } \\
\hline Rectus abdominis & $0.041[-0.224,0.301]$ & 0.761 \\
\hline Lateral abdominal wall & $-0.169[-0.414,0.098]$ & 0.213 \\
\hline Erector spinae & $-0.064[-0.321,0.202]$ & 0.639 \\
\hline Psoas major & $-0.363[-0.571,-0.111]$ & 0.006 \\
\hline Gluteus maximus & $-0.387[-0.590,-0.138]$ & 0.003 \\
\hline Quadriceps femoris & $0.040[-0.225,0.300]$ & 0.769 \\
\hline Hamstring & $-0.056[-0.314,0.210]$ & 0.679 \\
\hline Adductors & $-0.020[-0.281,0.244]$ & 0.884 \\
\hline Dorsiflexors & $0.170[-0.097,0.414]$ & 0.209 \\
\hline Plantar flexors & $-0.082[-0.338,0.185]$ & 0.548 \\
\hline
\end{tabular}

Bold values indicate significant correlations $(P<0.05)$ between CSA variables and personal best $100-\mathrm{m}$ sprint time in non-sprinters. In addition, the primary findings of this study showed that larger absolute and relative CSAs of the PM and GM correlated with better personal best $100 \mathrm{~m}$ sprint time in sprinters. Furthermore, a stepwise multiple regression analysis revealed that both CSAs of absolute PM and the relative GM were predictive variables of the personal best $100-\mathrm{m}$ sprint time. These findings suggest that the PM and GM may be specific muscles for superior sprint performance for sprinters.

With regard to the PM, it is known to be a major muscle for performing hip flexion because of the largest among the hip flexors [15]. Hoshikawa et al. [16] reported that larger PM CSA is correlated with higher hip flexor maximal torque. Moreover, using a computer simulation, Dorn et al. [17] determined that the PM (i.e., which combined the psoas major and iliacus) was the highest torque component for performing the hip flexion and contributed to rapidly accelerating the leg forward during sprinting. Furthermore, we and others previously reported that larger PM CSA is correlated with better sprint performance (e.g., personal best 100-m sprint time) in sprinters [7, 10-12]. Therefore, the present finding corroborates the results of these previous studies by showing positive correlations between absolute and relative PM CSAs and personal best 100-m sprint time in sprinters. The findings of our and other studies suggest that the PM is an important muscle for achieving superior sprint performance in sprinters.

With regard to the GM, it is known to be a major muscle for performing hip extension because of the largest among the hip extensors [4, 18]. Tayashiki et al. [19] reported a positive correlation between the GM thickness and hip extensor maximal torque, but this correlation did not reach significance. Moreover, Bartlett et al. [18] determined that an increase in the electromyographic activity of the GM is related to increased sprint velocity when sprinting. In addition, the GM plays an important role in decelerating the forward swing of the leg during the latter half of the swing phase and stabilizing the trunk against flexion while sprinting $[17,20]$. Furthermore, the computer simulation study, by Dorn et al. [17], determined that an increase in step frequency from a slow running to maximal sprinting is principally achieved by increasing the work of the hip extensor muscles, especially the GM, during the latter half of the swing phase. The increased step frequency is necessary to increasing sprint velocity during spirting $[12,21,22]$; thus, larger GM plays an important role in achieving better sprint performance, potentially by enhancing step frequency. Despite these previous findings, the relationship between GM size and sprint performance in sprinters remains poorly understood. Sugisaki et al. determined that greater 
muscle volume (MV) of the GM is correlated with better personal best $100 \mathrm{~m}$ sprint time in sprinters [10]. Therefore, the present finding corroborates their result by showing positive correlations between the absolute and relative GM CSAs and personal best $100 \mathrm{~m}$ sprint time in sprinters. Altogether, in addition to the PM, the GM is an important muscle for achieving superior sprint performance in sprinters.

In addition to the GM, the HAM, which is a biarticular muscle that extends the hip and flexes the knee, is known to be another major muscle among the hip extensors. The HAM is often considered a key muscle for achieving superior sprint performance in sprinters [23]. However, we and others previously reported the absence of relationship between the HAM CSA and sprint performance (e.g., personal best $100 \mathrm{~m}$ sprint time) in both groups of junior and adults sprinters $[7,9,12]$. Therefore, the present finding corroborates the results of these previous studies by showing no correlations between the absolute and relative HAM CSAs and personal best 100-m sprint time in adult sprinters. The findings of our and other studies suggest that the HAM may not be an important muscle for achieving superior sprint performance in sprinters.

\section{Limitations}

Although we used the CSAs for evaluating muscle size, it has been considered that the MV is a more reliable marker of muscle size than CSA [2]. Moreover, although we measured the CSAs of 10 selected muscles of the trunk and lower limb, number of muscles included in this study was relatively low when compared to that of several previous studies $[4,10]$. Furthermore, although we selected the PM and QF (i.e., the rectus femoris) as the hip flexors, it includes other agonist muscles such as the iliacus. Similarly, although we selected the GM and HAM as the hip extensors, it includes other synergistic muscles such as the gluteus minimus and gluteus medius. To further clarify the findings of the present study, further studies are needed to examine the relationships between MVs of all hip flexor and extensor muscles and sprint performance in sprinters.

\section{Abbreviations \\ CSA: Cross-sectional area; DF: Dorsiflexors; GM: Gluteus maximus; HAM: Hamstring; MRI: Magnetic resonance imaging; MV: Muscle volume; PM: Psoas major; QF: Quadriceps femoris.}

\section{Acknowledgements}

We are grateful to all subjects who gave of their time and effort to participate in this study.

\section{Authors' contributions}

NT and TS conceived and designed the experiment; NT TS YM RTTT MT and MO performed experiments; NT TS YM RT analyzed data; NT TS YM RT TT MT MO AN SF and TI interpreted results of experiments; NT and TS wrote the manuscript; MT MO AN SF and TI edited and revised manuscript. All authors have read and approved the manuscript.

\section{Funding}

This study was supported by the Japan Society for the Promotion of Science, Grants-in-Aid for Scientific Research (\#15K16497 to T.S; \#15H03077 to T.I).

Availability of data and materials

Data will be provided the corresponding author upon request.

\section{Ethics approval and consent to participate}

This study was approved by the Ethics Committee of Ritsumeikan University (BKC-IRB-2011-009). Informed written consent was obtained from all participants.

\section{Consent for publication \\ Not applicable.}

\section{Competing interests}

The authors declare that they have no competing interests.

\section{Author details}

${ }^{1}$ Research Organization of Science and Technology, Ritsumeikan University, 1-1-1 Nojihigashi, Kusatsu 525-8577, Shiga, Japan. ${ }^{2}$ Faculty of Sport and Health Science, Ritsumeikan University, 1-1-1 Nojihigashi, Kusatsu, Shiga 525-8577, Japan.

Received: 23 November 2020 Accepted: 16 February 2021

Published online: 25 February 2021

\section{References}

1. Novacheck TF. The biomechanics of running. Gait Posture. 1998;7(1):77-95.

2. Fukunaga T, Miyatani M, Tachi M, Kouzaki M, Kawakami Y, Kanehisa H. Muscle volume is a major determinant of joint torque in humans. Acta Physiol Scand. 2001;172(4):249-55.

3. Abe T, Kumagai K, Brechue WF. Fascicle length of leg muscles is greater in sprinters than distance runners. Med Sci Sports Exerc. 2000;32(6):1125-9.

4. Handsfield GG, Knaus KR, Fiorentino NM, Meyer CH, Hart JM, Blemker SS. Adding muscle where you need it: non-uniform hypertrophy patterns in elite sprinters. Scand J Med Sci Sports. 2017;27(10):1050-60.

5. Hoshikawa Y, Muramatsu M, lida T, li N, Nakajima Y, Kanehisa H. Sex differences in the cross-sectional areas of psoas major and thigh muscles in high school track and field athletes and nonathletes. J Physiol Anthropol. 2011:30(2):47-53.

6. Ema R, Sakaguchi M, Kawakami Y. Thigh and psoas major muscularity and its relation to running mechanics in sprinters. Med Sci Sports Exerc. 2018;50:2085-91.

7. Hoshikawa Y, Muramatsu M, lida T, Uchiyama A, Nakajima Y, Kanehisa H, et al. Influence of the psoas major and thigh muscularity on 100-m times in junior sprinters. Med Sci Sports Exerc. 2006;38(12):2138-43.

8. Kumagai K, Abe T, Brechue WF, Ryushi T, Takano S, Mizuno M. Sprint performance is related to muscle fascicle length in male 100-m sprinters. J Appl Physiol. 2000;88(3):811-6.

9. Sugisaki N, Kanehisa H, Tauchi K, Okazaki S, Iso S, Okada J. The relationship between 30-m sprint running time and muscle cross-sectional areas of the psoas major and lower limb muscles in male college short and middle distance runners. Int J Sport Health Sci. 2011:9:1-7.

10. Sugisaki N, Kobayashi K, Tsuchie H, Kanehisa H. Associations between individual lower-limb muscle volumes and 100-m sprint time in male sprinters. Int J Sports Physiol Perform. 2018;13(2):214-9. 
11. Tottori N, Kurihara T, Otsuka M, Isaka T. Relationship between lateral differences in the cross-sectional area of the psoas muscle and curve running time. J Physiol Anthropol. 2016;35:3.

12. Tottori N, Suga T, Miyake Y, Tsuchikane R, Otsuka M, Nagano A, et al. Hip flexor and knee extensor muscularity are associated with sprint performance in sprint-trained preadolescent boys. Pediatr Exerc Sci. 2018;30(1):115-23.

13. Miyake Y, Suga T, Otsuka M, Tanaka T, Misaki J, Kudo S, et al. The knee extensor moment arm is associated with performance in male sprinters. Eur J Appl Physiol. 2017;117(3):533-9.

14. Cohen J. A power primer. Psychol Bull. 1992;112(1):155-9.

15. Bogduk N, Pearcy M, Hadfield G. Anatomy and biomechanics of psoas major. Clin Biomech. 1992;7(2):109-19.

16. Hoshikawa Y, lida T, li N, Muramatsu M, Nakajima Y, Chumank K, et al. Cross-sectional area of psoas major muscle and hip flexion strength in youth soccer players. Eur J Appl Physiol. 2012;112(10):3487-94.

17. Dorn TW, Schache AG, Pandy MG. Muscular strategy shift in human running: dependence of running speed on hip and ankle muscle performance. J Exp Biol. 2012;215(11):1944-56.

18. Bartlett JL, Sumner B, Ellis RG, Kram R. Activity and functions of the human gluteal muscles in walking, running, sprinting, and climbing. Am J Phys Anthropol. 2014;153(1):124-31.
19. Tayashiki K, Hirata K, Ishida K, Kanehisa H, Miyamoto N. Associations of maximal voluntary isometric hip extension torque with muscle size of hamstring and gluteus maximus and intra-abdominal pressure. Eur J Appl Physiol. 2017;117:1267-72.

20. Lieberman DE, Raichlen DA, Pontzer $\mathrm{H}$, Bramble DM, Cutright-Smith E. The human gluteus maximus and its role in running. J Exp Biol. 2006;209(11):2143-55.

21. Morin JB, Gimenez P, Edouard P, Arnal P, Jimenez-Reyes P, Samozino P, et al. Sprint acceleration mechanics: The major role of hamstrings in horizontal force production. Front Physiol. 2015;6:404.

22. Weyand PG, Sternlight DB, Bellizzi MJ, Wright S. Faster top running speeds are achieved with greater ground forces not more rapid leg movements. J Appl Physiol. 2000;89(5):1991-9.

23. Delecluse $\mathrm{C}$. Influence of strength training on sprint running performance. Current findings and implications for training. Sports Med. 1997:24:147-56.

\section{Publisher's Note}

Springer Nature remains neutral with regard to jurisdictional claims in published maps and institutional affiliations.
Ready to submit your research? Choose BMC and benefit from:

- fast, convenient online submission

- thorough peer review by experienced researchers in your field

- rapid publication on acceptance

- support for research data, including large and complex data types

- gold Open Access which fosters wider collaboration and increased citations

- maximum visibility for your research: over $100 \mathrm{M}$ website views per year

At BMC, research is always in progress.

Learn more biomedcentral.com/submissions 